\title{
Erratum to: Stability of operational taxonomic units: an important but neglected property for analyzing microbial diversity
}

\author{
Yan He ${ }^{1}$, J. Gregory Caporaso ${ }^{2,3}$, Xiao-Tao Jiang ${ }^{1}$, Hua-Fang Sheng ${ }^{1}$, Susan M. Huse ${ }^{4}$, Jai Ram Rideout ${ }^{3}$, \\ Robert C. Edgar ${ }^{5}$, Evguenia Kopylova ${ }^{6}$, William A. Walters ${ }^{7}$, Rob Knight ${ }^{6,8}$ and Hong-Wei Zhou ${ }^{1 *}$
}

\section{Correction}

After publication of this work [1], we noted that one of the references in the introduction was not described properly. With respect to the original authors, we clarify that this sentence in introduction: "Due to the lack of a gold standard of 'correct' OTUs, several measurements have been used to evaluate the performance of clustering methods, for example, rationality of OTU structure [2, 3], computational efficiency (that is, runtime and memory requirements) [4], and the ability to cope with OTU inflation [5]" should be stated as "Due to the lack of a gold standard of 'correct' OTUs, several measurements have been used to evaluate the performance of clustering methods, for example, rationality of OTU structure [2], use of the Matthew's Correlation Coefficient [3], computational efficiency (that is, runtime and memory requirements) [4], and the ability to cope with OTU inflation [5]".

\section{Competing interests}

The authors declare that they have no competing interests.

\section{Authors' contributions}

$Y H, X T J, S M H, R K$, and HWZ conceived the method; JGC, JRR, RCE, and WAW implemented the software; YH, XTJ, JGC, HFS, SMH, and HWZ performed the experiments and analyzed the data; $\mathrm{YH}, \mathrm{SMH}, \mathrm{JGC}, \mathrm{JK}, \mathrm{EK}, \mathrm{RK}$, and HWZ wrote the manuscript. All authors read and approved the final manuscript.

\section{Author details}

'State Key Laboratory of Organ Failure Research, Department of Environmental Health, Guangdong Provincial Key Laboratory of Tropical Disease Research, School of Public Health and Tropical Medicine, Southern Medical University, Guangzhou 510515, China. ${ }^{2}$ Department of Biological Sciences, Northern Arizona University, PO Box 5640, Flagstaff, AZ, 86011-5640, USA. ${ }^{3}$ Center for Microbial Genetics and Genomics, Northern Arizona University, PO Box 4073, Flagstaff, AZ, 86011-4073, USA.
${ }^{4}$ Department of Pathology and Laboratory Science, Warren Alpert Medical School, Brown University, 70 Ship Street, Providence, RI, 02912, USA. ${ }^{5}$ Tiburon, CA, 94920, USA. ${ }^{6}$ Department of Pediatrics, University of California at San Diego, 9500 Gilman Drive MC0763, La Jolla, CA, 92093-0763, USA. ${ }^{7}$ Department of Molecular Biology and Genetics, Cornell University, 526 Campus Road, Ithaca, NY, 14853, USA. ${ }^{8}$ Department of Computer Science and Engineering, University of California at San Diego, 9500 Gilman Drive MC0763, La Jolla, CA, 92093-0763, USA.

Received: 22 July 2015 Accepted: 22 July 2015

Published online: 31 July 2015

\section{References}

1. He Y, Caporaso JG, Jiang XT, Sheng HF, Huse SM, Rideout JR, et al. Stability of operational taxonomic units: an important but neglected property for analyzing microbial diversity. Microbiome. 2015;3:20.

2. Jiang $X-T$, Zhang $H$, Sheng $H-F$, Wang $Y$, He $Y$, Zou F, et al. Two-Stage Clustering (TSC): a pipeline for selecting operational taxonomic units for the high-throughput sequencing of PCR amplicons. PLoS ONE. 2012;7.

3. Schloss PD, Westcott SL. Assessing and improving methods used in operational taxonomic unit-based approaches for 16S rRNA gene sequence analysis. Appl Environ Microbiol. 2011;77:3219-26.

4. Edgar RC. Search and clustering orders of magnitude faster than BLAST. Bioinformatics. 2010;26:2460-1.

5. Edgar RC. UPARSE: highly accurate OTU sequences from microbial amplicon reads. Nat Methods. 2013;10:996-8.

* Correspondence: biodegradation@gmail.com

${ }^{1}$ State Key Laboratory of Organ Failure Research, Department of

Environmental Health, Guangdong Provincial Key Laboratory of Tropical Disease Research, School of Public Health and Tropical Medicine, Southern Medical University, Guangzhou 510515, China

Full list of author information is available at the end of the article 